\title{
Damage Accumulation and Lifetime Prediction of Carbon Fiber Composite Pressure Vessels
}

M. Weber, B. Besancon, A. Bunsell, H. Zejli, Y. Favry, A. Thionnet

This document appeared in

Detlef Stolten, Thomas Grube (Eds.):

18th World Hydrogen Energy Conference 2010 - WHEC 2010

Parallel Sessions Book 4: Storage Systems / Policy Perspectives, Initiatives and Cooperations

Proceedings of the WHEC, May 16.-21. 2010, Essen

Schriften des Forschungszentrums Jülich / Energy \& Environment, Vol. 78-4

Institute of Energy Research - Fuel Cells (IEF-3)

Forschungszentrum Jülich $\mathrm{GmbH}$, Zentralbibliothek, Verlag, 2010

ISBN: 978-3-89336-654-5 


\title{
Damage Accumulation and Lifetime Prediction of Carbon Fiber Composite Pressure Vessels
}

\author{
Mathilde Weber, Brian Besancon, Air Liquide R\&D, Paris, France \\ Anthony Bunsell, Hasnae Zejli, Yves Favry, Alain Thionnet, Mines Paris Tech, \\ Paris, France
}

\section{Introduction}

The development of hydrogen as a reliable energy vector is strongly connected to the performance and level of safety of the components of the supply chain. In this respect, achieving an efficient storage is crucial to address transition markets and automotive markets.

For near-term markets, high pressure compressed gas storage in carbon fiber composite pressure vessels is currently the most promising technology. These pressure vessels are made of a plastic or metallic liner wrapped with carbon fibers embedded in a polymer matrix. This technology is currently industrialized for working pressures in the range of 200 to 300 bar (self-contained breathing apparatus and compressed natural gas storage for transportation). The feasibility and storage efficiency were demonstrated at higher working pressure (700 bar) in recent developments (European project Storhy for instance [1]).

However, challenges remain to improve performance and reliability of these pressure vessels while still ensuring the safety of cylinders in service over periods of 15 to 20 years. In order to avoid overdesign design and conservative use, a fundamental understanding of damage mechanisms and degradation of carbon fiber composite materials is required. Knowledge of the damage accumulation kinetics permits the assessment of residual lifetimes, which can be used to define design parameters such as the safety factor and during periodic inspections in order to determine whether a cylinder remains fit for service.

The composite structure of a pressure vessel is obtained by filament winding. The fibers are placed on geodesic paths around a mandrel, which later serves as a liner to ensure gas tightness. This means that when the vessel is pressurized the fibers are subjected only to tensile forces and at the level of the fibers an analogy can be made with the behavior of a unidirectional composite loaded in the fiber direction. The fibers support all but one percent of the mechanical stress in the walls of the pressure vessels and their failure is what determines the failure of these composite pressure vessels. As with a unidirectional composite, failure of the material means that the fibers have to break. Carbon fibers are perfectly elastic. In a bundle of fibers, there is a scattering of tensile strength and the tensile strength distribution follow Weibull statistics [2]. Weibull statistics are based on a view of failure processes which concludes that strength is not an intrinsic property of the material but rather a stochastic process governed by the probability of defects occurring within the sample tested [2].

This paper addresses the rate of damage accumulation in carbon fiber composites specimens and pressure vessels and more specifically of carbon fiber breaks accumulation. 
In a first part, a model developed in the framework of previous study is described. In a second part, the fiber break accumulation measurement by acoustic emission on samples and pressure vessels is discussed.

\section{Carbon Fiber Composites Failure Modeling}

The goal of the model discussed here is to simulate the failure of fibers under static load in the fibre direction. A finite element multi-scale approach has been adopted by Bunsell and co. $[3,4]$. The micromechanics study considers a three-dimensional analysis of a representative volume element (RVE) comprising all the processes to be considered. These are fiber strength and its stochastic nature, fiber tensile elastic modulus and the properties of the matrix. It is necessary to take into account interfacial debonding of the interface between the broken fiber and the matrix as well as considering the viscoelastic nature of the matrix so that the properties of the composites are time dependent, as are the effects of load transfer between neighboring fibers, which are due to shear of the matrix.

The model has been used in a previous study [4] to predict the failure of unidirectional plate specimens loaded in the fiber direction. It has been shown that the model accurately predicts tensile failure to within $\pm 2 \%$ and that the scatter of composite properties also can be simulated [4]. The model reveals that, in the absence of stress concentrations due to the geometry of the composite specimen, fiber breaks initially occur randomly within a unidirectional composite loaded in the fiber direction. These random breaks reflect the random nature of the defects on the fibers. Initially the breaks are isolated in the composite because they are embedded in the matrix material. However, as loading becomes more critical the breaks begin to cluster due to the stress concentrating effects induced by the shear of the matrix around the breaks. This leads to the failure of some neighboring fibers. When the clusters of breaks reach a critical size the composite becomes unstable and this will eventually lead to failure of the composite.

The same reference explores the effects of the viscoelastic properties of the matrix on the long term behavior of the composite under a steady load as shown in figure 1 . The model is capable of predicting the scatter of rates of fiber failures when compared to experimental curves obtained by acoustic emission as shown in figure 1 (comparison of 3 samples: 1,2 and 3 to simulations). Figure 1 also illustrates that fiber breaks will continue to occur, if the composite is held at a constant load, due to the relaxation of the matrix around earlier breaks, but at a decreasing rate. However if eventually several fibers break in the same region this will lead to clustering of the breaks and an acceleration of the damage. A physical instability arises when the density of fiber breaks reaches a critical threshold and the regions of damage begin to interfere. This behavior is predicted by the model [5] and has been observed experimentally [6] both on samples and pressure vessels. 


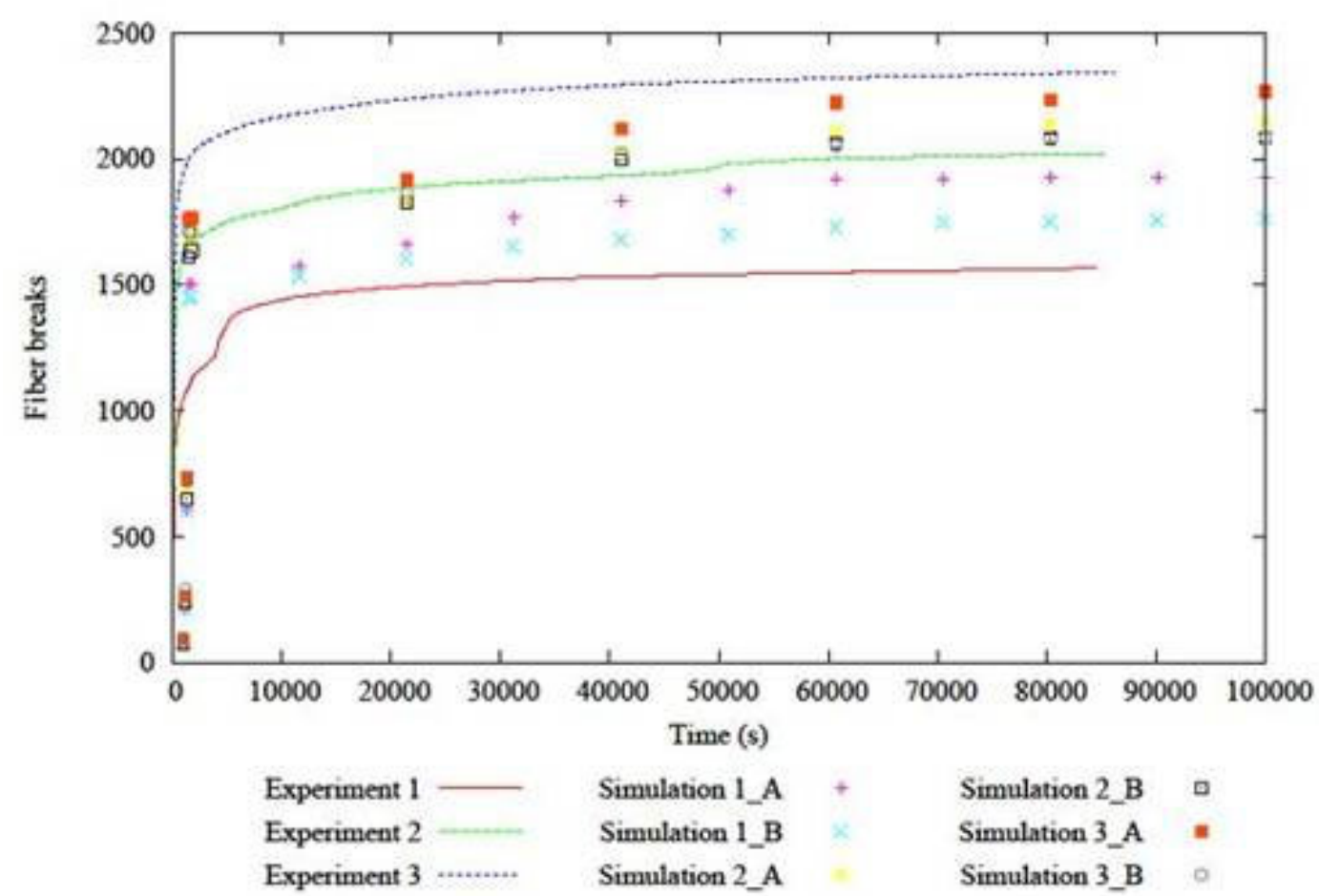

Figure 1: Comparison of simulated and experimental results of constant load tests, at $80 \%$ failure stress, with unidirectional CFRP (carbon fiber reinforced polymer) specimens loaded in the fiber direction [4].

\section{Damage Detection in Carbon Fiber Composites}

To compare experiments to the model, we need an experimental technique that can measure fiber breaks occurrence. In this study, acoustic emission is investigated. When subjected to static or cyclic load, a composite material will exhibit various types of damage mechanisms including fiber breaks as presented in Figure 2. This damages occurrence generates acoustic emissions; the main issue is to be able to discriminate fiber break from other damage mechanisms.

In a first step, plate specimen with various fiber orientations chosen so as to control the failure processes have been studied and the means of discriminating these processes using the acoustic emission technique have been determined. Six types of composite plate samples were investigated including pure epoxy resin, carbon fiber bundles (representative of the one used by cylinder manufacturer, Young modulus $=235 \mathrm{GPa}),\left[0^{\circ}\right] 4$ and $\left[90^{\circ}\right] 4$ coupons, [02/902]s and [902/02]s cross-plied coupons. 

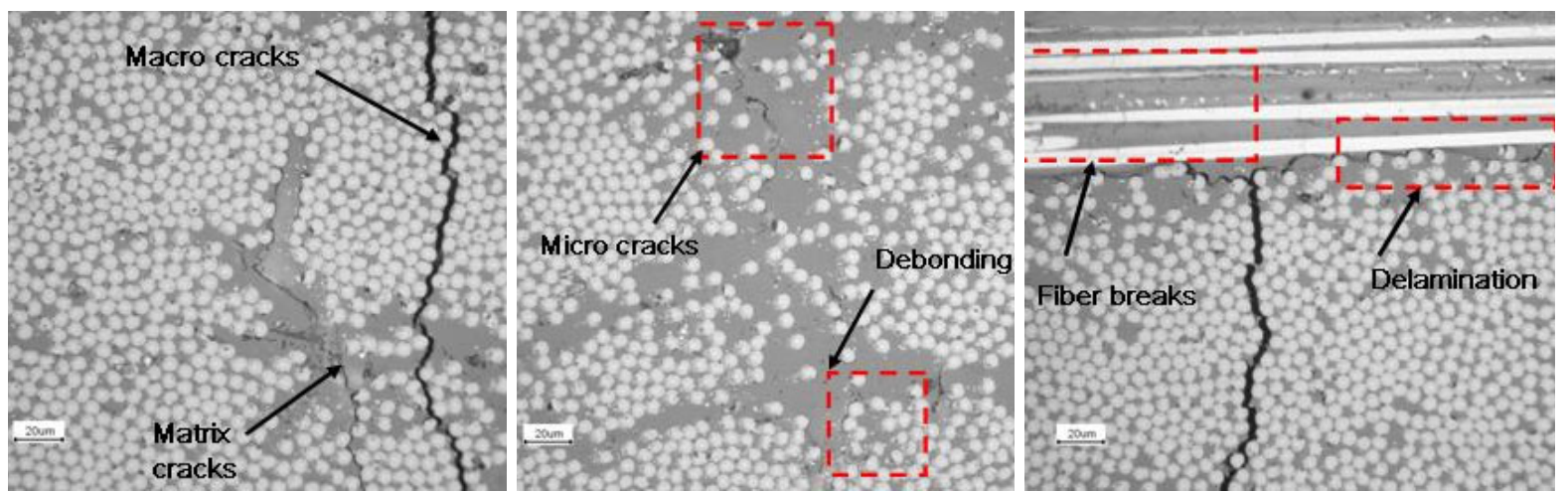

Figure 2: Failure processes in composites that generate acoustic emissions.

Details on the acoustic emission system and measurements are provided elsewhere [6]. A neural type network analysis of several parameters in parallel was carried out so as to better discriminate the types of emissions. It was possible to discriminate fiber breaks from other damages both on samples and on the pressure vessels tested in this study.

The pressure vessels studied were designed to have a burst strength of $115 \mathrm{MPa}$ and were subjected to pressures up to $60 \mathrm{MPa}$. In order to examine the effects of cyclic pressurization, the pressure vessel was subjected to an initial pressurization up to $60 \mathrm{MPa}$ and held at that pressure for twenty four hours; it was then cycled one hundred times from near to zero to $60 \mathrm{MPa}$ and again held at the maximum pressure for twenty four hours. This was repeated after a further 1000 cycles, then 5000 cycles and finally 15,000 cycles. During the 20 hours periods at $60 \mathrm{MPa}$, the acoustic emissions were monitored. The results are displayed in figure 3.

As with the plate specimens, after the first cycle of pressurization, emissions were only recorded near to the maximum pressure. Figure 3 reveals that the major part of the emissions came from fiber breaks and other processes were secondary. It can be seen that the cycles of pressure did not alter the overall curve of damage accumulation which would have been obtained by holding the pressure constant [6,7, see example figure 1]. The conclusion is that the ageing process in the composite wrap on the pressure vessel is controlled by the viscoelastic nature of the composite, as described in the above model and not significantly affected by cycling the pressure. 

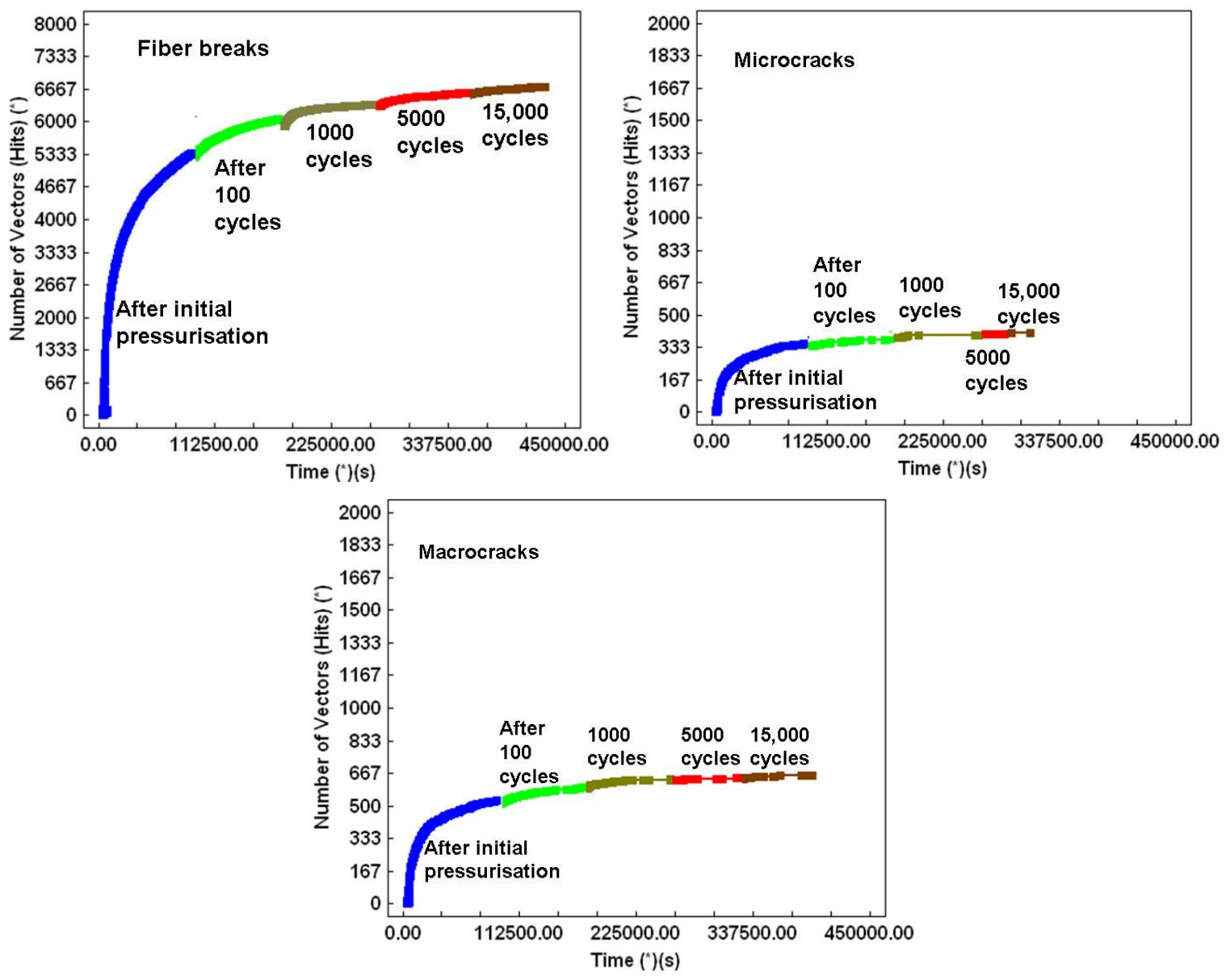

Figure 3: The evolution of damage mechanisms as the pressure vessel was repeatedly subjected to pressure and then held at the maximum pressure of 60MPa for periods of twenty four hours.

\section{Conclusion}

In this study, the state of damage accumulation in carbon fiber composites specimens and pressure vessels subjected to static and cycling loading has been measured by acoustic emission. Multi-variable analysis of the acoustic emission data has been used to identify the relevant damage mechanisms contributing to composite failure as well as its kinetics.

Failure of the composite pressure vessel is controlled by the progressive failure of the reinforcing fibers which at first is randomly distributed in the composite but if allowed to progress sufficiently will lead to clustering of fiber breaks and instability of the pressure vessel. Fiber breaks occur due to load transfer through the matrix and delayed breaks occur because of the viscoelastic nature of the matrix which leads to its relaxation around earlier breaks and subsequent overloading of intact fibers in regions of fiber breaks. This mechanism is confirmed by finite element modeling. Based on these data, lifetime prediction will be carried out. 


\section{References}

[1] S Colom, M Weber and F Barbier, Storhy: A European development of composite vessels for 70MPa Hydrogen storage, World Hydrogen Energy Conference (2008)

[2] A.R.Bunsell 'A Handbook of Textile and Technical Fibres' Woodhead Publisher and CRC, Cambridge 2009

[3] S. Blassiau, A. Thionnet and A.R. Bunsell ,Composite structures, 74 (2006) 303-318

[4] S. Blassiau, A. Thionnet and A.R. Bunsell, Composite structures, 83 (2008) 312-323.

[5] S. Camara, A 3D 'Multiscale Finite Element Analysis of Damage Accumulation in Carbon Fiber Unidirectional Composites: Applied to Pressure Vessels': MS thesis Nebraska University Lincoln, 2009.

[6] K.Heng-Yi, H.Zejli, A.Thionnet and A.R.Bunsell. To be published.

[7] A.R.Bunsell, Reinforced Plastics February (2006) 39-41. 